Chirurg $2021 \cdot 92: 128-133$

https://doi.org/10.1007/s00104-021-01354-4

Angenommen: 7. Januar 2021

Online publiziert: 28. Januar 2021

(c) Springer Medizin Verlag GmbH, ein Teil von Springer Nature 2021

\author{
Jochen Dutzmann ${ }^{1}$ Andrej Michalsen ${ }^{2}$ \\ ' Universitätsklinik und Poliklinik für Innere Medizin III, Universitätsklinikum Halle (Saale), Halle (Saale), \\ Deutschland \\ ${ }^{2}$ Klinik für Anästhesiologie, Intensivmedizin, Notfallmedizin und Schmerztherapie, Klinikum Konstanz, \\ Konstanz, Deutschland
}

\title{
„Triage-Empfehlungen“ der Fachgesellschaften
}

\section{Hintergrund, Zusammenfassung und Kommentar}

\section{Hintergrund}

Angesichts dramatischer Szenen in der von der COVID-19-Pandemie besonders betroffenen Stadt Bergamo und ohne Zeit für eine kritische gesellschaftliche Auseinandersetzung veröffentlicht die Società Italiana di Anestesia Analgesia Rianimazione e Terapia Intensiva (SIAARTI) am 06.03.2020 die europaweit ersten Triage-Empfehlungen „Clinical Ethics Recommendations for the Allocation of Intensive Care Treatments in exceptional, resource-limited circumstances“. Eine vergleichbar deletäre Situation wie in Bergamo erschien auch Experten in Deutschland möglich, zumal mit einer Verbreitung der Infektion unter deutschen Urlaubsrückkehrern aus dem österreichischen Skiort Ischgl sowie bereits Ende Februar 2020 im Rahmen einer Karnevalsveranstaltung im nordrhein-westfälischen Kreis Heinsberg erste rasche und unkontrollierte Ausbrüche auch diesseits der Landesgrenzen $\mathrm{zu}$ erleben waren. Unter Federführung der Akademie für Ethik in der Medizin e.V. (AEM) und der Deutschen Interdisziplinären Vereinigung für Intensiv- und Notfallmedizin (DIVI) erarbeiten Intensivmediziner von 9 Fachgesellschaften gemeinsam mit Vertretern aus Medizinethik und Jurispru-

\section{Leitlinie}

Marckmann G, Neitzke G, Schildmann J et al. (2020) Med Klin Intensivmed Notfmed 115:477-485. https://doi.org/10.1007/s00063020-00708-w denz klinisch-ethische Empfehlungen zur „Entscheidung über die Zuteilung intensivmedizinischer Ressourcen im Kontext der COVID-19-Pandemie“, die in einer ersten Version am 26.03.2020 online veröffentlicht und zur Diskussion gestellt und nach kritischer Einarbeitung der Kommentare am 16.04.2020 in einer zweiten Version veröffentlicht werden. Während mit den vorhandenen und neu geschaffenen intensivmedizinischen Kapazitäten die erste Pandemiewelle deutschlandweit nahezu flächendeckend gut bewältigt werden kann und es zunächst den Anschein hat, als bedürfte es keiner praktischen Anwendung der Empfehlung, ächzt das Gesundheitssystem schon bald unter der zweiten, ungleich heftigeren Welle, sodass die „Triage-Frage" droht, akut zu werden. Der Begriff "Triage“ wird üblicherweise in Kriegsund Katastrophenszenarien verwendet und stößt mitunter auch auf Ressentiments. Insofern kann besser der Begriff „Priorisierung“ verwendet werden. Die vorgestellten Empfehlungen behandeln aber genau die gleiche schwierige Herausforderung: die Verteilung knapper Ressourcen in Krisenzeiten.

\section{Allgemeine Grundsätze der Entscheidungsfindung}

Die Empfehlung betont, dass ärztliche Indikation und Patientenwille Grundlage jeder patientenzentrierten Entscheidung darstellen. Dabei ist eine Intensivtherapie grundsätzlich nicht indiziert, wenn
- die Therapie als medizinisch aussichtslos eingeschätzt wird, weil keine Besserung oder Stabilisierung erwartet wird, oder

- ein Überleben an den dauerhaften Aufenthalt auf der Intensivstation gebunden wäre.

Patienten, die eine intensivmedizinische Therapie ablehnen, werden ebenfalls nicht intensivmedizinisch behandelt. Grundlage hierfür kann der aktuelle, vorausverfügte (z. B. in Form einer Patientenverfügung), früher geäußerte oder mutmaßliche Wille sein.

Nur wenn die Ressourcen nicht ausreichen - weder im eigenen Haus noch regional oder überregional - muss unausweichlich entschieden werden, welche intensivpflichtigen Patienten intensivmedizinisch behandelt und welche nicht (oder nicht mehr) intensivmedizinisch behandelt werden sollen. Ausschließlich in einer solchen Situation wird eine Einschränkung der sonst gebotenen patientenzentrierten Behandlungsentscheidung erforderlich, und es muss - analog der Triage in der Katastrophenmedizin über die Verteilung der begrenzt verfügbaren Ressourcen entschieden werden. Die dann erforderlichen Priorisierungen müssen nach transparenten, medizinisch und ethisch gut begründeten Kriterien erfolgen und ausdrücklich nicht in der Absicht, Menschen oder Menschenleben $\mathrm{zu}$ bewerten, sondern mit der Zielsetzung, mit den (begrenzten) Ressourcen möglichst vielen Patienten eine Teilha- 


\section{Patientenetikett}

\section{Datum/Uhrzeit}

Notwendigkeit einer intensivmedizinischen Therapie

\section{Teammitglieder (Name/Funktion)}

Klinische Ethik beteiligt? $\quad$ ja $\square$ /nein $\square$

\section{Notwendigkeit einer intensivmedizinischen Therapie}

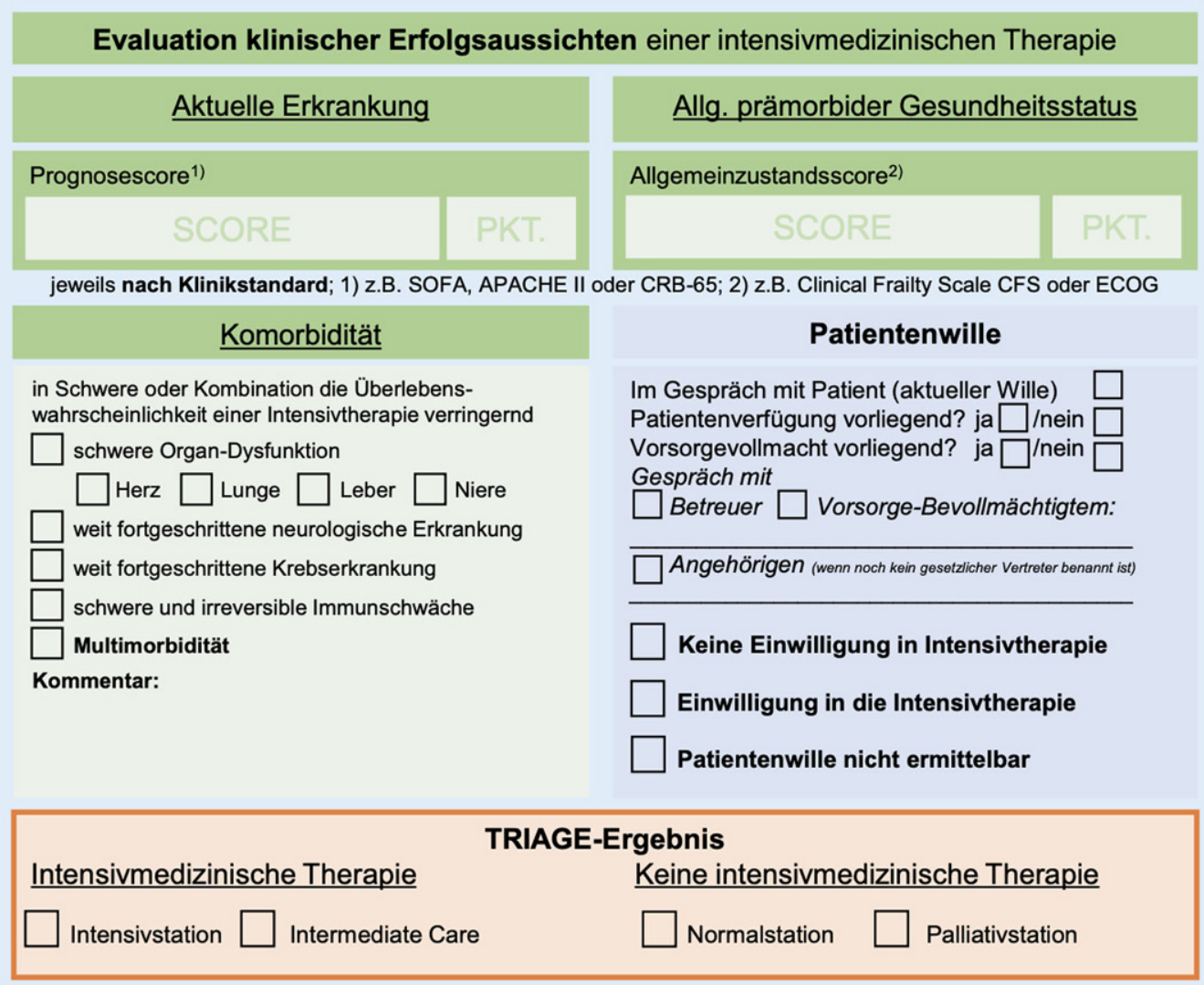

\section{RE-EVALUATION am Datum/Uhrzeit:}

Einschätzung des klinischen Verlaufs:

Fortsetzung oder Therapiezieländerung, weil:
Abb. $1<$ Dokumentationshilfe zur Priorisierung bei Ressourcenknappheit. SOFA Sepsis-related Organ Failure Assessment, APACHE II Acute Physiology and Chronic Health Disease Classification System II, CRB-65 Confusion, Respiratory rate, Blood pressure, ECOG Eastern Cooperative Oncology Group 
be an der medizinischen Versorgung unter Krisenbedingungen zu ermöglichen. Die Priorisierung soll sich daher am Kriterium der klinischen Erfolgsaussicht orientieren. Eine Priorisierung ist aufgrund des Gleichheitsgebots nicht vertretbar nur innerhalb der Gruppe der COVID-19-Erkrankten und nicht zulässig aufgrund des kalendarischen Alters, aufgrund sozialer Merkmale oder aufgrund bestimmter Grunderkrankungen. Damit ist eine Benachteiligung bestimmter Subpopulationen, z.B. von Menschen mit Behinderung, ausdrücklich nicht statthaft. Zugleich unterliegen aber Subpopulationen auch derselben Einschätzung betreffend die klinische Erfolgsaussicht, dürfen also auch nicht bevorzugt werden.

\section{Verfahren und Kriterien für Priorisierungsentscheidungen bei Ressourcenknappheit}

In der klinischen Praxis können unterschieden werden:

- Entscheidungen, bei welchen Patienten intensivmedizinische Maßnahmen begonnen werden (Ex-anteTriage),

- Entscheidungen, bei welchen Patienten bereits eingeleitete intensivmedizinische Maßnahmen beendet werden (Ex-post-Triage).

Es ist sicherzustellen, dass eine angemessene (Weiter-)Behandlung für diejenigen Patienten zur Verfügung steht, die nicht oder nicht mehr intensivmedizinisch behandelt werden können, einschließlich einer ggf. erforderlichen Palliativversorgung.

Ein vorab definiertes Verfahren der Entscheidungsfindung mit klar geregelten Verantwortlichkeiten ist Voraussetzung für konsistente, faire sowie medizinisch und ethisch gut begründete Priorisierungsentscheidungen. Daher sollen die Entscheidungen möglichst nach dem Mehr-Augen-Prinzip erfolgen unter Beteiligung

- von möglichst 2 intensivmedizinisch erfahrenen Ärzten einschließlich Primär- und Sekundärbehandler beteiligter Fachgebiete,

Chirurg 2021 · 92:128-133 https://doi.org/10.1007/s00104-021-01354-4

(c) Springer Medizin Verlag GmbH, ein Teil von Springer Nature 2021

\section{J. Dutzmann · A. Michalsen \\ „Triage-Empfehlungen" der Fachgesellschaften. Hintergrund, Zusammenfassung und Kommentar}

\section{Zusammenfassung}

Bei Triage-Entscheidungen handelt es sich um die Verteilung knapper Ressourcen in Krisenzeiten weshalb der Begriff üblicherweise in Kriegs- und Katastrophenszenarien verwendet wird. Unter Federführung der Akademie für Ethik in der Medizin e.V. (AEM) und der Deutschen Interdisziplinären Vereinigung für Intensiv- und Notfallmedizin (DIVI) erarbeiten Intensivmediziner von 9 Fachgesellschaften gemeinsam mit Vertretern aus Medizinethik und Jurisprudenz klinisch-ethische Empfehlungen zur „Entscheidung über die Zuteilung intensivmedizinischer Ressourcen im Kontext der COVID-19-Pandemie", die in einer ersten Version am 26.03.2020 online veröffentlicht und zur Diskussion gestellt und nach kritischer Einarbeitung der Kommentare am 16.04.2020 in einer zweiten Version veröffentlicht werden. In der nun stattfindenden zweiten Welle der COVID-19Pandemie droht die Triage-Frage, akut zu werden.

Schlüsselwörter

Corona · Pandemie · SARS-CoV-2 · COVID-19. Priorisierung

\section{Triage recommendations of the specialist societies. Background, summary and comments}

\section{Abstract}

Triage decisions are concerned with the distribution of limited resources in times of crises, which is why the term is normally used in war and catastrophe scenarios. Under the leadership of the Academy for Ethics in Medicine (AEM) and the German Interdisciplinary Association for Intensive Care and Emergency Medicine (DIVI), intensive care physicians from nine specialist societies together with representatives from medical ethics and jurisprudence elaborated clinical and ethical recommendations on "necision on the allocation of intensive medical care resources in the context of the COVID-19 pandemic". These recommendations were published online in a first version on 26 March 2020 and comments were requested. After a critical consideration of the comments a second version was published on 16 April 2020. In the currently occurring second wave of the COVID-19 pandemic, the question of triage now threatens to become acute.

Keywords

Corona · Pandemic · SARS-CoV-2 · COVID-19. Prioritization
- von möglichst einem erfahrenen Vertreter der Pflegenden,

- ggf. von weiteren Fachvertretern (z. B. klinische Ethik).

Nach Möglichkeit sollen die Entscheidungen im Konsens getroffen werden. Zum Umgang mit Dissens sollten die jeweiligen Kliniken angemessene Vorgehensweisen festlegen. Die Entscheidungen sollten in den berufs- und fachgruppenübergreifenden Teams getroffen, transparent gegenüber Patienten, Angehörigen (soweit möglich) und ggf. rechtlichen Stellvertretern kommuniziert und sachgerecht dokumentiert werden (• Abb. 1).
Die Schritte der Entscheidungsfindung und die dabei anzuwendenden Kriterien werden in der klinisch-ethischen Empfehlung als Ablaufschema dargestellt (•Abb. 2). Neben dem Schweregrad der aktuellen Erkrankung spielen Anzahl und Ausmaß der vorliegenden Begleiterkrankungen in der Bewertung der Erfolgsaussicht neben einer Vielzahl weiterer klinischer Parameter eine wesentliche Rolle.

\section{Juristische Einordnung}

Die Empfehlungen weisen explizit darauf hin, dass aus verfassungsrechtlichen Gründen Menschenleben nicht gegen Menschenleben abgewogen werden dür- 
Interprofessionelles Mehr-Augen-Prinzip

möglichst 2 intensivmedizinisch erfahrenen Ärzte inkl. Primär-und Sekundärbehandler

+ möglichst Vertreter der Pflege und bei Bedarf weitere Disziplinen (z.B. klinische Ethik)

$\downarrow$

Schritt 1:

Besteht intensivmedizinische Behandlungsnotwendigkeit?

\section{NEIN}

\section{JA}

\section{Schritt 2:}

Besteht eine realistische klinische Erfolgsaussicht einer Intensivtherapie zum aktuellen Zeitpunkt?

\section{JA}

\section{Schritt 3:}

Liegt die Einwilligung des Patienten vor (aktuell, vorausverfügt, zuvor mündlich geäußert oder mutmaßlich)?

$$
\checkmark \text { JA (oder Patientenwille nicht ermittelbar) }
$$

Schritt 4: Priorisierung im Mehr-Augen-Prinzip nach Prüfung von Kriterien für den Therapieerfolg und der Ressourcen

\section{Kriterien für geringe Erfolgsaussicht bei Initial- oder Re-Evaluation}

\section{Aktuelle Erkrankung}

Höherer Schweregrad

z.B. akutes Lungenversagen

(ARDS, Acute Respiratory

Distress Syndrome)

Begleitende akute Organversagen z.B. anhand des SOFA-Scores ermittelt

Ggf. prognostischer Marker für COVID-19-Patienten

\section{Komorbiditäten}

Vorhandensein schwerer Komorbiditäten, wenn diese in ihrer Schwere oder Kombination die Überlebenswahrscheinlichkeit bei einer Intensivtherapie erheblich verringern, z.B.:

- schwere Organ-Dysfunktion

- weit fortgeschrittene neurologische Erkrankung

- weit fortgeschrittene Krebserkrankung

- schwere, irreversible Immunschwäche

- Multimorbidität

Allgemeiner Gesundheitsstatus

Erhöhte Gebrechlichkeit (z.B. Clinical Frailty Scale [CFS])

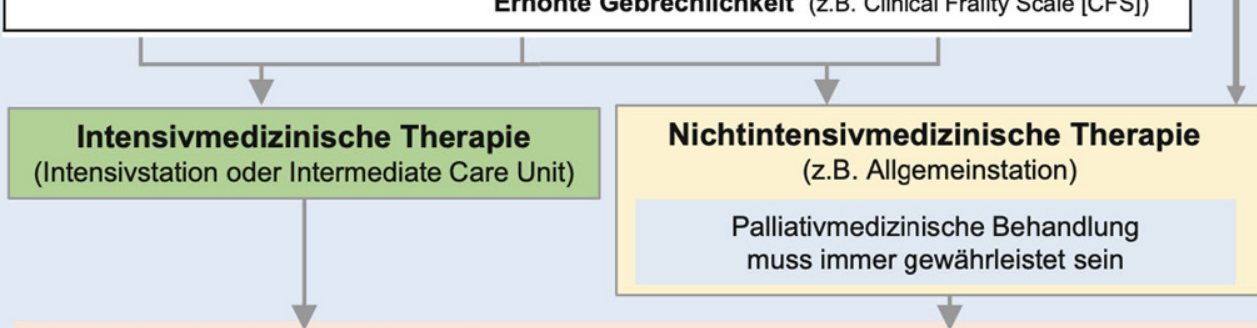

Re-Evaluation Intensivtherapie in angemessenen Abständen und in jedem Falle bei

klinisch relevanten Veränderungen der klinischen Erfolgsaussicht sowie

Änderungen des Verhältnisses von Bedarf und zur Verfügung stehenden Ressourcen

\section{Voraussetzungen für die Beendigung einer Intensivtherapie bei Re-Evaluation}

- Fortsetzung der Intensivtherapie widerspricht dem (erklärten oder mutmaßlichen) Patientenwillen

- Therapieziel ist nicht mehr realistisch erreichbar

- Behandlungsversuch ist nach Ablauf eines Beobachtungszeitraums mit zuvor festgelegten Kriterien ohne Erfolg

- Fortschreitendes Multiorganversagen
Abb. $2<$ Ablaufschema bei nicht ausreichenden Intensivressourcen. SOFA Sepsis-related Organ Failure Assessment fen, gleichzeitig jedoch Behandlungsressourcen verantwortungsbewusst eingesetzt werden müssten. Dabei beruhte die Empfehlung mit dem Ziel, die Anzahl vermeidbarer Todesfälle zu minimieren, auf den nach Einschätzung der Verfas- ser am ehesten begründbaren ethischen Grundsätzen in einer tragischen Entscheidungssituation. Die Verfasser weisen darauf hin, dass eine abschließende juristische Einordnung nicht Gegenstand der Empfehlung sei. Ein Eilantrag auf eine verbindliche rechtliche Regelung der Triage wurde durch das Bundesverfassungsgericht u.a. mit Verweis auf die Unwahrscheinlichkeit des tatsächlichen Eintretens einer Triage-Situation am 14.08.2020, also vor Beginn der zweiten 
Infektionswelle, abgelehnt. Eine verfassungsrechtliche Bewertung einer solchen tragischen Entscheidungssituation steht damit weiterhin aus. Sowohl in einer am 27.03.2020 veröffentlichten Ad-hoc-Stellungnahme des Deutschen Ethikrats als auch in der inzwischen geführten Diskussion unter Rechtswissenschaftlern wird insbesondere die Rechtmäßigkeit der Ex-post-Triage durchaus kritisch gesehen. Grundsätzlich gilt allerdings auch in Zeiten knapper Ressourcen, dass ausschließlich Behandlungsteams Indikationsstellungen und die Einschätzung der klinischen Erfolgsaussicht und daraus folgend - Therapieziel und Behandlungsumfang der je individuellen Patienten bestimmen können - nicht Juristen oder Interessenverbände.

\section{Fazit für die Praxis}

- Bei Triage-Entscheidungen handelt es sich um die Verteilung knapper Ressourcen in Krisenzeiten.

- Wenn die Ressourcen nicht ausreichen, muss entschieden werden, welche intensivpflichtigen Patienten intensivmedizinisch behandelt und welche nicht (oder nicht mehr) intensivmedizinisch behandelt werden sollen.

- Die Priorisierung soll sich am Kriterium der klinischen Erfolgsaussicht orientieren.

- Die Entscheidungen sollten möglichst nach dem Mehr-Augen-Prinzip erfolgen.

- Eine verfassungsrechtliche Bewertung einer solchen tragischen Entscheidungssituation steht weiterhin aus.

\section{Korrespondenzadresse}

\section{Dr. med. Jochen Dutzmann}

Universitätsklinik und Poliklinik für Innere Medizin III, Universitätsklinikum Halle (Saale) Ernst-Grube-Str. 40, 06120 Halle (Saale), Deutschland

jochen.dutzmann@uk-halle.de

Dr. med. Andrej Michalsen, M.P.H./Univ. of Texas

Klinik für Anästhesiologie, Intensivmedizin, Notfallmedizin und Schmerztherapie, Klinikum Konstanz

Mainaustr. 35, 78464 Konstanz, Deutschland andrejmichalsen@hotmail.de

\section{Einhaltung ethischer Richtlinien}

Interessenkonflikt. J. Dutzmann und A. Michalsen haben als Koautoren an den hier diskutierten klinischethischen Empfehlungen mitgewirkt.

Für diesen Beitrag wurden von den Autoren keine Studien an Menschen oder Tieren durchgeführt. Für die aufgeführten Studien gelten die jeweils dort angegebenen ethischen Richtlinien.
Hier steht eine Anzeige. Springer 
Hier steht eine Anzeige.

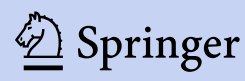

